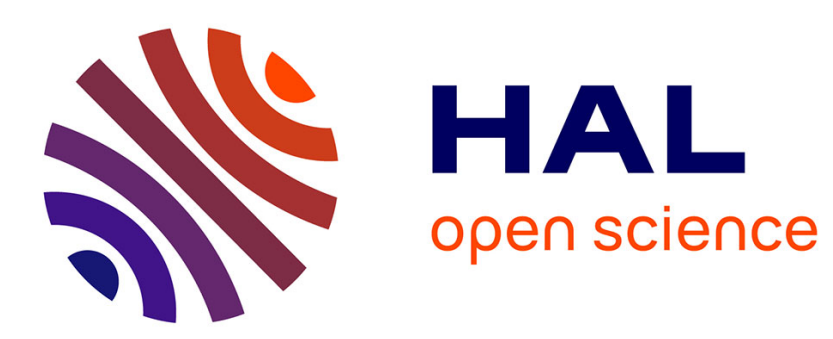

\title{
The wave-front tracking algorithm for Hughes' model of pedestrian motion
}

Paola Goatin, Matthias Mimault

\section{To cite this version:}

Paola Goatin, Matthias Mimault. The wave-front tracking algorithm for Hughes' model of pedestrian motion. SIAM Journal on Scientific Computing, 2013, 35 (3), pp.B606-B622. hal-00751260

\author{
HAL Id: hal-00751260 \\ https://hal.inria.fr/hal-00751260
}

Submitted on 13 Nov 2012

HAL is a multi-disciplinary open access archive for the deposit and dissemination of scientific research documents, whether they are published or not. The documents may come from teaching and research institutions in France or abroad, or from public or private research centers.
L'archive ouverte pluridisciplinaire HAL, est destinée au dépôt et à la diffusion de documents scientifiques de niveau recherche, publiés ou non, émanant des établissements d'enseignement et de recherche français ou étrangers, des laboratoires publics ou privés. 


\title{
THE WAVE-FRONT TRACKING ALGORITHM FOR HUGHES' MODEL OF PEDESTRIAN MOTION*
}

\author{
PAOLA GOATIN AND MATTHIAS MIMAULT ${ }^{\dagger}$
}

\begin{abstract}
We code the wave-front tracking algorithm for Hughes' model of pedestrian motion with generalized running cost. This model displays a non-classical dynamic at the splitting point between the two directions of motion. The wave-front tracking scheme provides us with reference solutions to test numerically the convergence of classical finite volume schemes, which do not treat explicitly the dynamics at the turning point.
\end{abstract}

Key words. Macroscopic pedestrian flow models, conservation laws, eikonal equation, wavefront tracking, finite volume schemes.

AMS subject classifications. 35L65

1. Introduction. We consider a generalization of the macroscopic pedestrian flow model introduced by Hughes [9]. The pedestrian density $\rho=\rho(t, x)$ is assumed to evolve according to the following system consisting in a scalar conservation law expressing mass conservation coupled with an eikonal equation giving the preferred direction depending on the density profile, which in the one dimensional case read:

$$
\begin{aligned}
& \rho_{t}-\left(\rho v(\rho) \frac{\phi_{x}}{\left|\phi_{x}\right|}\right)_{x}=0, \\
& \left|\phi_{x}\right|=c(\rho),
\end{aligned}
$$

in the spatial domain $\Omega=]-1,1[$. The above equations must be completed with homogeneous (weak) Dirichlet boundary conditions at $x= \pm 1$, which represent the exits locations

$$
\begin{aligned}
& \rho(t,-1)=\rho(t, 1)=0, \\
& \phi(t,-1)=\phi(t, 1)=0,
\end{aligned}
$$

and an initial density profile $\rho(0, \cdot)=\rho_{0} \in \mathrm{BV}(\mathbb{R})$.

Above, $x \in \Omega$ is the space variable, $t \geq 0$ is the time, $\rho=\rho(t, x) \in[0,1]$ is the (normalized) crowd density, $c=c(\rho)$ is the cost function, $v(\rho)=(1-\rho)$ is the mean velocity and we set $f(\rho)=\rho v(\rho)=\rho(1-\rho)$. We assume that $c:[0,1[\rightarrow[1,+\infty[$ is a smooth function such that $c(0)=1$ and $c^{\prime}(\rho) \geq 0$ for $\rho \in[0,1[$. Recall that the literature usually proposes the choice

$$
c(\rho)=\frac{1}{v(\rho)}
$$

as in [4, 9, 10].

We observe that (1.1a) can be rewritten as

$$
\rho_{t}-\left(f(\rho) \operatorname{sgn}\left(\phi_{x}\right)\right)_{x}=0,
$$

\footnotetext{
${ }^{*}$ This research was supported by the ERC Starting Grant 2010 under the project "TRAffic Management by Macroscopic Models"

${ }^{\dagger}$ INRIA Sophia Antipolis - Méditerranée, 2004 route des Lucioles - BP 93, 06902 Sophia Antipolis Cedex, France (paola.goatin@inria.fr and matthias.mimault@inria.fr)
} 
and the unique viscosity solution to the Dirichlet problem $1.1 \mathrm{~b}, 1.2 \mathrm{~b}$ is given by the value function of the corresponding control problem with discontinuous coefficient $c(\rho)$, i.e.

$$
\phi(t, x)= \begin{cases}\int_{-1}^{x} c(\rho(t, y)) d y & \text { if } x \leq \xi(t), \\ \int_{x}^{1} c(\rho(t, y)) d y & \text { if } x \geq \xi(t),\end{cases}
$$

where $\xi(t)$ is implicitly defined by the identity

$$
\int_{-1}^{\xi(t)} c(\rho(t, y)) d y=\int_{\xi(t)}^{1} c(\rho(t, y)) d y .
$$

Therefore, equation 1.1a can be written as a scalar conservation law with discontinuous space-time dependent flux:

$$
\rho_{t}+F(t, x, \rho)_{x}=0,
$$

where $F(t, x, \rho)=\operatorname{sgn}(x-\xi(t)) f(\rho)$. Observe that the position of the discontinuity is not a priori fixed, as assumed in previous results on conservation laws with discontinuous fluxes (see for example [12] and references therein), but depends non-locally on $\rho$ itself. Therefore, known convergence results for finite volume schemes do not apply in this setting.

From the mathematical point of view, problem 1.1 presents a non trivial coupling between a scalar conservation law and an eikonal equation, that poses several challenging questions concerning existence, uniqueness, numerical approximation and construction of the solutions. Some preliminary results concerning properties of solutions have been obtained recently in 1, 5. In particular, they provide a notion of entropy weak solutions, a discussion on their qualitative behavior, and an algorithm to construct locally in time a self-similar solution in a neighborhood of the turning point $x=\xi(t)$ (see [1, Theorem 1]).

Here we are interested in computing numerically the weak solutions of the (nonregularized) hyperbolic problem (1.1). As remarked above, classical finite volume techniques for discontinuous fluxes do not apply here due to the fact that the discontinuity location is not a-priori fixed, but depends non-locally on the solution itself. Nevertheless, it appears that a direct application of classical shemes allows to recover the exact solutions. Since these are not known explicitly, the convergence of the finite volume schemes is numerically tested on accurate approximations of entropy weak solutions computed using the wave-front algorithm (for a detailed review of the method we refer to [7]).

2. Basic properties of solutions. For sake of simplicity, we refer to the initialboundary value problem

$$
\begin{array}{ll}
\rho_{t}+F(t, x, \rho)_{x}=0, & x \in \Omega, t>0, \\
\left|\phi_{x}\right|=c(\rho), & x \in \Omega \\
\rho(0, x)=\rho_{0}(x), & \\
\rho(t,-1)=\rho(t, 1)=0, & t>0, \\
\phi(t,-1)=\phi(t, 1)=0, &
\end{array}
$$


Following [5], we look for the following class of solutions.

DeFinition 2.1. (Entropy Weak Solutions).

A function $\rho \in \mathbf{C}^{\mathbf{0}}\left(\mathbb{R}^{+} ; \mathbf{L}^{\mathbf{1}}(\Omega)\right) \cap \mathrm{BV}\left(\mathbb{R}^{+} \times \Omega ;[0,1]\right)$ is an entropy weak solution of the initial-boundary value problem (2.1) if the following Kružkov-type entropy inequality holds for all $k \in[0,1]$ and all test functions $\psi \in \mathbf{C}_{\mathbf{c}}^{\infty}\left(\mathbb{R} \times \Omega ; \mathbb{R}^{+}\right)$:

$$
\begin{aligned}
0 \leq & \int_{0}^{+\infty} \int_{-1}^{1}\left(|\rho-k| \psi_{t}+\Phi(t, x, \rho, k) \psi_{x}\right) d x d t+\int_{-1}^{1}\left|\rho_{0}(x)-k\right| \psi(0, x) d x \\
& +\operatorname{sgn}(k) \int_{0}^{+\infty}(f(\rho(t, 1-))-f(k)) \psi(t, 1) d t \\
& +\operatorname{sgn}(k) \int_{0}^{+\infty}(f(\rho(t,-1+))-f(k)) \psi(t,-1) d t \\
& +2 \int_{0}^{+\infty} f(k) \psi(t, \xi(t)) d t .
\end{aligned}
$$

Above, $\Phi(t, x, \rho, k)=\operatorname{sgn}(\rho-k)(F(t, x, \rho)-F(t, x, k))$ and the boundary conditions are intended in weak form as in 2]. In particular, the traces of the solution at the boundary points must satisfy

$$
\begin{array}{cl}
f(\rho(t,-1+)) \geq f(k), & \text { for all } k \in[0, \rho(t,-1+)], \\
f(\rho(t, 1-)) \geq f(k), & \text { for all } k \in[0, \rho(t, 1-)] .
\end{array}
$$

This in particular implies $\rho(t,-1+) \leq 1 / 2$ and $\rho(t, 1-) \leq 1 / 2$.

Besides, by taking suitable test functions $\psi$ vanishing along $x=\xi(t)$, it is easy to recover the Rankine-Hugoniot condition

$$
f\left(\rho^{+}\right)+f\left(\rho^{-}\right)=\dot{\xi}(t)\left(\rho^{+}-\rho^{-}\right),
$$

where we have denoted by $\rho^{+}=\rho^{+}(t)=\rho(t, \xi(t)+)$ and $\rho^{-}=\rho^{-}(t)=\rho(t, \xi(t)-)$ the right and left traces of $\rho$ at $x=\xi(t)$. We remark here that if $\rho(t, \cdot)$ is continuous at $x=\xi(t)$, then 2.3 gives $f(\rho(t, \xi(t)))=0$ and therefore $\rho(t, \xi(t)) \in\{0,1\}$.

For further properties of entropy weak solutions, such as maximum principle, we refer the reader to [5].

3. The Riemann solver at the turning point. The argument proposed in [1] is based on the observation that, due to finite wave propagation speed, for every fixed $\bar{t} \geq 0$ the solution $\rho(t, x)$ is well defined away from the turning point $\bar{\xi}=\xi(\bar{t})$ for $t>\bar{t}$ sufficiently small, say on $[-1, \bar{\xi}-\delta) \cup(\bar{\xi}+\delta, 1]$ for some $\delta>0$. Therefore, the following quantity is well defined

$$
\left\{\int_{\bar{\xi}+\delta}^{1}-\int_{-1}^{\bar{\xi}-\delta}\right\} \partial_{t}[c(\rho(t, y))] d y=: \Psi^{*} .
$$

Moreover, let us assume that the the function $\rho(\bar{t}, x)$ is constant in a left and right neighbors of $\bar{\xi}$, let's say

$$
\rho(\bar{t}, x)= \begin{cases}\rho_{L} & \text { if } \bar{\xi}-2 \delta<x<\bar{\xi}, \\ \rho_{R} & \text { if } \bar{\xi}<x<\bar{\xi}+2 \delta .\end{cases}
$$

Assuming $\Psi^{*}$ to be constant for small times $t>\bar{t}$, the solution around the turning point will be self-similar, behaving as a solution of a Riemann problem. This assumption is met when there are no interacting patterns in the whole interval $[-1,1]$. 
Therefore, for small times $t>\bar{t}$ we have

$$
\Psi[\rho]=\Psi^{*}+\left\{\int_{\xi(t)}^{\bar{\xi}+\delta}-\int_{\bar{\xi}-\delta}^{\xi(t)}\right\} \partial_{t}[c(\rho(t, y))] d y .
$$

The term $\Psi^{*}$ depends on $\rho(\bar{t}, \cdot)$ in a non-local way and may range all over $\mathbb{R}$, independently of $\rho_{L}$ and $\rho_{R}$. The solution around $\bar{\xi}$ will be classified according to the value of $\Psi^{*}$.

Theorem 3.1. (Riemann Solver at $\bar{\xi}=\xi(\bar{t})$ ). Assume that (3.1) and (3.2) hold, then a self-similar solution around $(\bar{t}, \bar{\xi})$ is defined locally in time as follows:

1. If $\rho_{L}>\rho_{R}$, the following cases occur.

1a) If

$$
\Psi *<\frac{f\left(\rho_{R}\right)+f\left(\rho_{L}\right)}{\rho_{R}-\rho_{L}}\left[c\left(\rho_{R}\right)+c\left(\rho_{L}\right)\right],
$$

then there exists a unique intermediate value $\rho_{M}$, with $\rho_{L}>\rho_{M}>$ $\rho_{R}$, such that the solution is given by the turning curve $\xi$ followed by a rarefaction between $\rho_{M}$ and $\rho_{R}$.

1b) If

$$
\begin{array}{r}
\frac{f\left(\rho_{R}\right)+f\left(\rho_{L}\right)}{\rho_{R}-\rho_{L}}\left[c\left(\rho_{R}\right)+c\left(\rho_{L}\right)\right] \leq \Psi * \\
\leq-v\left(\rho_{L}\right)\left(1+c\left(\rho_{L}\right)\right)-v\left(\rho_{R}\right)\left(1-c\left(\rho_{R}\right)\right),
\end{array}
$$

then a unique intermediate value $\rho_{M} \in\left[0, \rho_{R}\right]$ exists, such that the solution is given by the turning curve $\xi$ followed by a shock between $\rho_{M}$ and $\rho_{R}$. If the equality holds in the r.h.s. of (3.4), then $\rho_{M}=0$ : vacuum appears between the turning point and the shock.

1c) If

$$
\begin{array}{r}
-v\left(\rho_{L}\right)\left(1+c\left(\rho_{L}\right)\right)-v\left(\rho_{R}\right)\left(1-c\left(\rho_{R}\right)\right)<\Psi * \\
<v\left(\rho_{R}\right)\left(1+c\left(\rho_{R}\right)\right)+v\left(\rho_{L}\right)\left(1-c\left(\rho_{L}\right)\right),
\end{array}
$$

then the solution is given by a shock of speed $\rho_{L}-1$ followed by $\xi$ and by a shock of speed $1-\rho_{R}$, the intermediate state around $\xi$ being $\rho_{M}=0$.

1d) Finally if

$$
\Psi * \geq v\left(\rho_{R}\right)\left(1+c\left(\rho_{R}\right)\right)+v\left(\rho_{L}\right)\left(1-c\left(\rho_{L}\right)\right),
$$

then the solution is given by a shock between $\rho_{L}$ and $\rho_{M} \in\left[0, \rho_{R}\right]$ followed by $\xi$. If the equality holds in (3.6), then $\rho_{M}=0$, otherwise $\rho_{M}>0$.

2. If $\rho_{L}<\rho_{R}$, the following cases occur.

2a) If

$$
\Psi * \leq-v\left(\rho_{L}\right)\left(1+c\left(\rho_{L}\right)\right)-v\left(\rho_{R}\right)\left(1-c\left(\rho_{R}\right)\right),
$$

then a unique intermediate value $\rho_{M} \in\left[0, \rho_{L}\right]$ exists, such that the solution is given by $\xi$ followed by a shock between $\rho_{M}$ and $\rho_{R}$. If the equality holds in (3.7), then $\rho_{M}=0$ : vacuum appears between the turning point and the shock. 
2b) If

$$
\begin{array}{r}
-v\left(\rho_{L}\right)\left(1+c\left(\rho_{L}\right)\right)-v\left(\rho_{R}\right)\left(1-c\left(\rho_{R}\right)\right)<\Psi * \\
<v\left(\rho_{R}\right)\left(1+c\left(\rho_{R}\right)\right)+v\left(\rho_{L}\right)\left(1-c\left(\rho_{L}\right)\right),
\end{array}
$$

then the solution is given by a shock of speed $\rho_{L}-1$ followed by $\xi$ and by a shock of speed $1-\rho_{R}$, the intermediate state being $\rho_{M}=0$.

2c) If

$$
\begin{array}{r}
v\left(\rho_{R}\right)\left(1+c\left(\rho_{R}\right)\right)+v\left(\rho_{L}\right)\left(1-c\left(\rho_{L}\right)\right) \leq \Psi * \\
\leq \frac{f\left(\rho_{R}\right)+f\left(\rho_{L}\right)}{\rho_{R}-\rho_{L}}\left[c\left(\rho_{R}\right)+c\left(\rho_{L}\right)\right],
\end{array}
$$

then the solution is given by a shock between $\rho_{L}$ and $\rho_{M} \in\left[0, \rho_{L}\right]$, followed by the turning curve $\xi$. If equality holds in the l.h.s. of $(3.9)$, then $\rho_{M}=0$, otherwise $\rho_{M}>0$.

2d) Finally if

$$
\Psi *>\frac{f\left(\rho_{R}\right)+f\left(\rho_{L}\right)}{\rho_{R}-\rho_{L}}\left[c\left(\rho_{R}\right)+c\left(\rho_{L}\right)\right],
$$

then there exists a unique intermediate value $\rho_{M}$, with $\rho_{R}>\rho_{M}>$ $\rho_{L}$, such that the solution is given by a rarefaction between $\rho_{L}$ and $\rho_{M}$ followed by the turning curve $\xi$.

3. If $\rho_{L}=\rho_{R}=\rho^{*}$, the following cases occur.

3a) If

$$
\Psi * \leq-2 v\left(\rho^{*}\right),
$$

then a unique intermediate value $\rho_{M} \in\left[0, \rho^{*}\right]$ exists, such that the solution is given by the turning curve $\xi$ followed by a shock between $\rho_{M}$ and $\rho_{R}$. If the equality holds in (3.11), then $\rho_{M}=0$ : vacuum appears between the turning point and the shock.

3b) If

$$
-2 v\left(\rho^{*}\right)<\Psi *<2 v\left(\rho^{*}\right)
$$

then the solution is given by a shock of speed $\rho^{*}-1$ followed by $\xi$ and by a shock of speed $1-\rho^{*}$, the intermediate state across $\xi$ being $\rho_{M}=0$.

3c) Finally if

$$
\Psi * \geq 2 v\left(\rho^{*}\right),
$$

then the solution is given by a shock between $\rho_{L}$ and $\rho_{M} \in\left[0, \rho_{R}\right]$ followed by $\xi$. If equality holds in (3.13), then $\rho_{M}=0$, otherwise $\rho_{M}>0$.

The proof follows closely the one of [1, Theorem 1] and it is therefore omitted. 


\section{Numerical schemes.}

4.1. The wave-front tracking scheme. The wave-front tracking technique for classical scalar conservation laws consists in constructing piece-wise constant exact solutions of an approximated problem with piecewise constant initial datum and piecewise linear flux $f^{\nu}$, coinciding with $f=\rho v(\rho)$ on the mesh $\mathcal{M}_{\nu}=\left\{\rho_{i}^{\nu}\right\}_{i=0}^{2^{\nu}} \subset \Omega$ defined by

$$
\mathcal{M}_{\nu}=\left(2^{-\nu} \mathbb{N} \cap[0,1]\right)
$$

for $\nu \in \mathbb{N}, \nu>0$. We refer the reader to [3, 7] for further details. We describe here the extension of the method to the case of the coupled problem (1.1).

Let $\rho_{0}^{\nu}$ be a piecewise constant function defined by

$$
\rho_{0}^{\nu}=\sum_{j \in \mathbb{Z}} \rho_{0, j}^{\nu} \chi_{] x_{j-1}, x_{j}\right]} \quad \text { with } \rho_{0, j}^{\nu} \in \mathcal{M}_{\nu}
$$

which approximates $\rho_{0}$ in the sense of the strong $\mathbf{L}^{\mathbf{1}}$ topology, that is

$$
\lim _{\nu \rightarrow \infty}\left\|\rho_{0}^{\nu}-\rho_{0}\right\|_{\mathbf{L}^{1}(\Omega)}=0,
$$

and such that $\operatorname{TV}\left(\rho_{0}^{\nu}\right) \leq \operatorname{TV}\left(\rho_{0}\right)$. Above, we set $x_{0}=\xi_{0}$, which is calculated from the cost balance equation (1.6) at $t=0$ :

$$
\int_{-1}^{\xi_{0}} c\left(\rho_{0}^{\nu}(y)\right) d y=\int_{\xi_{0}}^{1} c\left(\rho_{0}^{\nu}(y)\right) d y
$$

that reduces to

$$
\sum_{j \leq 0} \rho_{0, j}^{\nu}\left(x_{j}-x_{j-1}\right)=\sum_{j>0} \rho_{0, j}^{\nu}\left(x_{j}-x_{j-1}\right)
$$

Observe that we may have $\rho_{0,0}^{\nu}=\rho_{0,1}^{\nu}$.

For small times $t>0$, a piecewise approximate solution $\left(\rho^{\nu}, \xi_{\nu}\right)$ to 2.1 is constructed piecing together the solutions to the Riemann problems

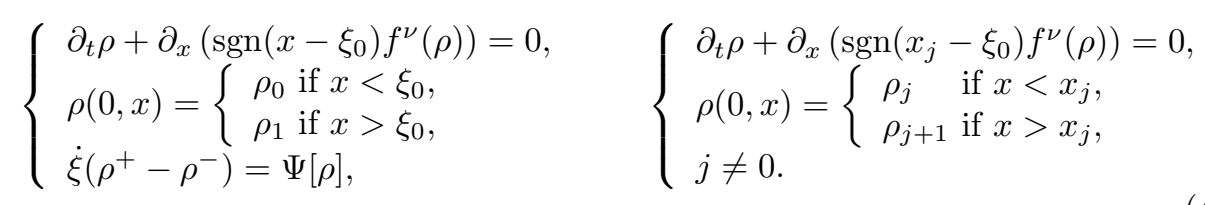

Solving the problem by front tracking replaces the regular rarefaction fronts by rarefaction fans of constant values $\rho_{j}^{\nu}, j=l_{1}, \ldots, l_{N_{\nu}}$, such that $\left|\rho_{j}^{\nu}-\rho_{j-1}^{\nu}\right|=2^{-\nu}$, separated by jump discontinuities moving with speeds $\lambda_{j}=1-\rho_{j}^{\nu}-\rho_{j-1}^{\nu}$, see Fig. 4.1 . Note that the solution to the Riemann problem in (4.1), left, is constructed by means of the Riemann solver described previously in Theorem 3.1, where the theoretic value of the intermediate state $\rho_{M}$ is approximated by the closest point of the mesh $\mathcal{M}_{\nu}$. This introduces an error in the resolution of the linearized problem, but guarantees that the approximate solution keeps lying on $\mathcal{M}_{\nu}$. The (piece-wise constant) speed of the approximated turning curve $\xi^{\nu}$ is then computed using the Rankine-Hugoniot equation 2.3. 


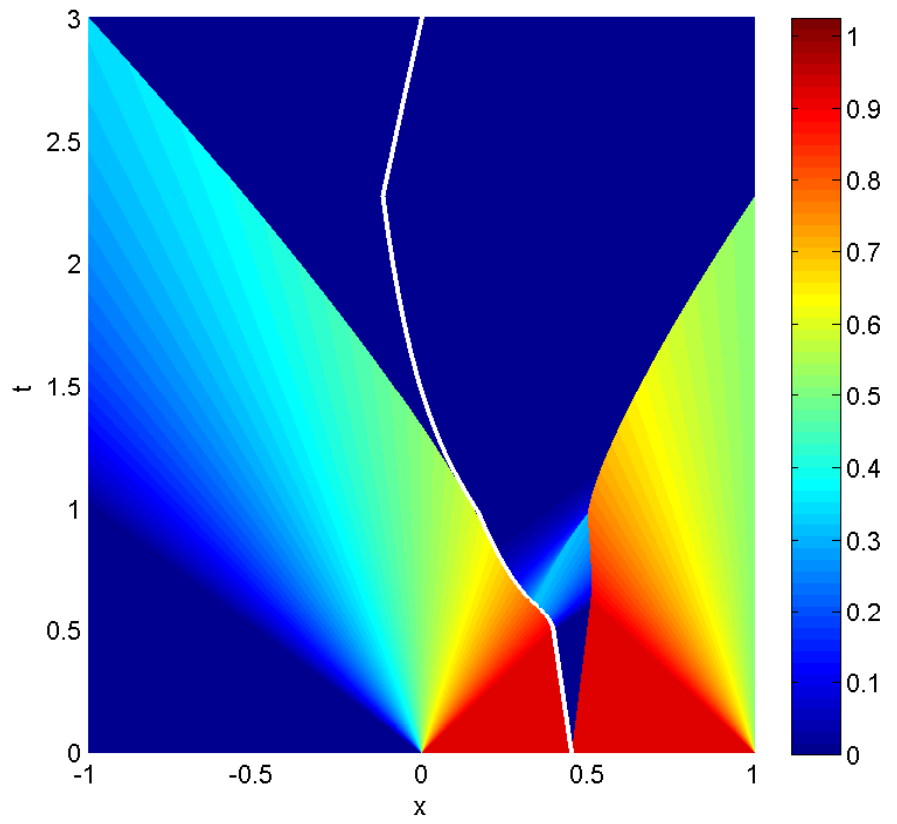

FIG. 4.1. Wave-front tracking solution of (2.1) with $\rho_{0}(x)=0$ for $\left.x \in\right]-1,0\left[\right.$ and $\rho_{0}(x)=0.9$ for $x \in] 0,1\left[\right.$, and mesh size $\Delta \rho=2^{-10}$. The white curve is the trajectory $x=\xi(t)$.

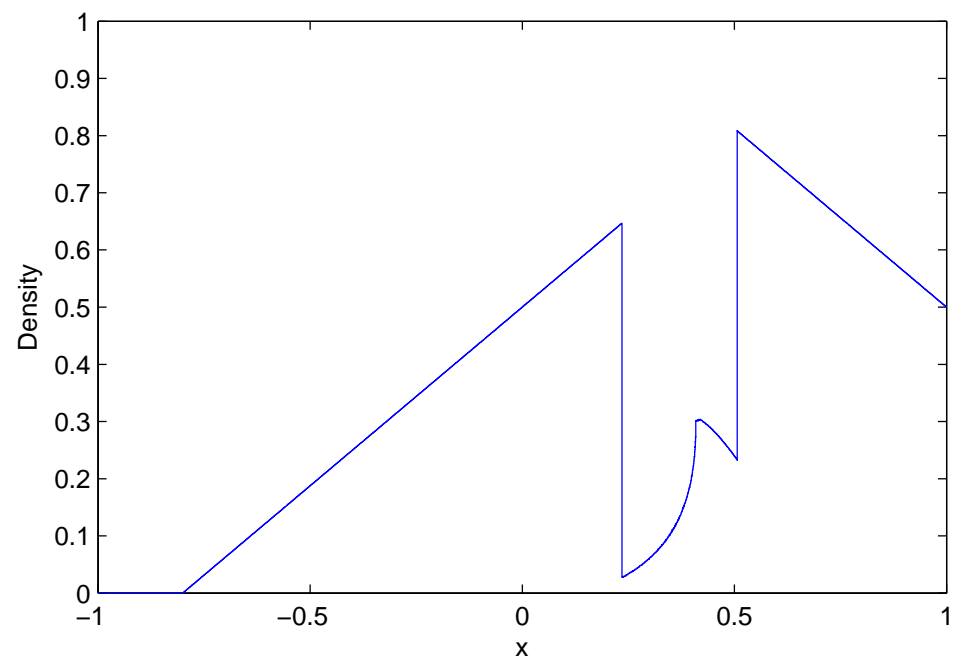

FIG. 4.2. Density profile at time $t=0.8$ with initial density $\rho_{0}(x)=0$ for $\left.x \in\right]-1,0[$ and $\rho_{0}(x)=0.9$ for $\left.x \in\right] 0,1\left[\right.$, computed using wave-front tracking scheme with mesh size $\Delta \rho=2^{-10}$. The position of the turning point $x=\xi(t)$ coincides with the first jump discontinuity from the left. 
The piecewise constant approximate solution $\rho^{\nu}$ constructed above can be prolonged up to the fist time $\bar{t}>0$, where two discontinuities collide, or a discontinuity hits the turning curve $\xi^{\nu}$. In both cases, a new Riemann problem arises and its solution, obtained in the former case with the classical Riemann solver and in the latter by means of Theorem 3.1, allows to extend $\rho^{\nu}, \xi^{\nu}$ further in time. We point out that the absorbing boundary conditions $1.2 \mathrm{a}$ are taken into account by simply dropping the waves hitting the left and right boundaries.

This procedure will provide us with a reference solution to numerically test the convergence of classical finite volume schemes. Even if the theoretical convergence of wave-front tracking approximations to exact solutions of 2.1) makes the object of on-going research, numerical evidence of this convergence can be found in the tests reported in Section 5.2 .

The Matlab code used for the numerical tests presented in Section 5 can be downloaded at the following URL:

http://www-sop.inria.fr/members/Paola.Goatin/wft.html.

4.2. Finite volume schemes. In this section, we describe the algorithm used in [5] for numerical simulations of the model (1.1). Finite volume schemes are commonly used to compute numerically solutions of the $2 \mathrm{D}$ version of the model, see for example [8, 11] and references therein for more details and results.

Here we aim at investigating the behavior of classical schemes near the turning curve, and whether they manage to capture correctly the behavior of the solution constructed via the wave-front tracking algorithm.

Given an initial datum $\rho(0, x)=\rho_{0}(x)$ and homogeneous Dirichlet boundary conditions $(1.2)$, we solve (1.1) in an iterative manner at each time step, i.e.

1. Given $\rho$, solve the eikonal equation $1.1 \mathrm{~b}$ by the fast sweeping method, see Section 4.2.1.

2. Given $\phi$, solve the non-linear conservation law (1.1a) using Godunov or Rusanov scheme.

The domain $[-1,1]$ is divided into $N$ uniform cells $I_{j}=\left[x_{j-1 / 2}, x_{j+1 / 2}\right]$ with centers at points $x_{j}=j \Delta x$, with $\Delta x=2 / N$. The explicit algorithm used to generate the approximations $\rho_{j}^{n}$ was introduced by Towers in [14] and is written in conservation form

$$
\rho_{j}^{n+1}=\rho_{j}^{n}-\frac{\Delta t^{n}}{\Delta x}\left(k_{j+\frac{1}{2}}^{n} \mathrm{~h}_{j+\frac{1}{2}}^{n}-k_{j-\frac{1}{2}}^{n} \mathrm{~h}_{j-\frac{1}{2}}^{n}\right),
$$

where $\Delta t^{n}=t^{n+1}-t^{n}$ is chosen to satisfy the following CLF condition

$$
\Delta t^{n}<0.5 \frac{\Delta x}{\max \left\{\max _{j}\left|f^{\prime}\left(\rho_{j}^{n}\right)\right|,\left|\dot{\xi}^{n}\right|\right\}} .
$$

In the above formula, the coefficient 0.5 is chosen to avoid interactions of $\xi$ with the cell boundaries, and $|\dot{\xi}|$ is estimated at each time step by deriving the implicit expression (1.6), which gives

$$
\dot{\xi}(t)\left(c\left(\rho^{-}\right)+c\left(\rho^{+}\right)\right)=-\int_{-1}^{\xi(t)} c(\rho(t, y))_{t} d y+\int_{\xi(t)}^{1} c(\rho(t, y))_{t} d y .
$$

From the above identity we recover the (sharp) upper bound

$$
\left|\dot{\xi}^{n}\right| \leq \frac{1}{2}\left|\sum_{j}\left(1-\rho_{j}^{n}-\rho_{j+1}^{n}\right)\left(c\left(\rho_{j}^{n}\right)-c\left(\rho_{j+1}^{n}\right)\right)\right| .
$$


In 4.2, we set $k_{j \pm 1 / 2}^{n}=\operatorname{sgn}\left(\phi_{x}^{n}\left(x_{j \pm 1 / 2}\right)\right)$, where

$$
\phi_{x}^{n}\left(x_{j+1 / 2}\right) \simeq \frac{\phi^{n}\left(x_{j+1}\right)-\phi^{n}\left(x_{j}\right)}{\Delta x} .
$$

The numerical flux $\mathrm{h}_{j+1 / 2}^{n}=h\left(\rho_{j}^{n}, \rho_{j+1}^{n}\right)$ is chosen to be monotone and consistent, i.e. $h(\rho, \rho)=f(\rho)=\rho v(\rho)$. In order to maintain the monotonicity of the scheme, we transpose the arguments when $k_{j+1 / 2}$ changes sign, i.e.

$$
\mathrm{h}_{j+\frac{1}{2}}= \begin{cases}h\left(\rho_{j}, \rho_{j+1}\right) & \text { if } k_{j+\frac{1}{2}} \geq 0, \\ h\left(\rho_{j+1}, \rho_{j}\right) & \text { if } k_{j+\frac{1}{2}}<0 .\end{cases}
$$

\subsubsection{The Fast Sweeping algorithm [15].}

Discretization. The method uses a Godunov upwind difference scheme to discretize the partial differential equation at interior points of the domain:

$$
\left[\left(\phi_{j}^{h}-\phi_{x \min }^{h}\right)^{+}\right]^{2}=c\left(\rho_{i}\right)^{2} h^{2}, \quad j=2, \ldots, N-1,
$$

where $\phi_{j}^{h} \simeq \phi\left(x_{j}\right)$ and $\phi_{x m i n}^{h}=\min \left(\phi_{j-1}^{h}, \phi_{j+1}^{h}\right)$ and

$$
(x)^{+}= \begin{cases}x, & x>0 \\ 0, & x \leq 0\end{cases}
$$

Initialization. We assign exact values at boundary grid points $x= \pm 1$ to enforce boundary condition $\phi( \pm 1)=0$. We assign sufficiently large positive values at all other grid points. These points will be updated later.

Iterations. At each grid point $x_{j}$ whose value is not fixed during the initialization, compute the solution of (4.4), denoted by $\bar{\phi}$, from the current values of its neighbors $\phi_{j \pm 1}^{h}$ and then update $\phi_{j}^{h}$ to be the smaller one between $\bar{\phi}$ and $\phi_{j}^{h}$, i.e. $\phi_{j}^{\text {new }}=$ $\min \left(\phi_{j}^{\text {old }}, \bar{\phi}\right)$. We sweep the whole domain with two alternating orderings repeatedly:

$$
\text { (1) } j=1: N, \quad \text { (2) } j=N: 1 \text {, }
$$

and we stop when the fixed error threshold is met.

4.2.2. Godunov scheme. The Godunov scheme [6] for a conservation law of type $u_{t}+F(u)_{x}=0$ is classically obtained by using the exact solution with piecewise constant initial data. The numerical flux is $h(u, v)=F(\mathcal{R}(0 ; u, v))$, where $\mathcal{R}(0 ; u, v)$ is the solution of the Riemann problem with left and right states $u$ and $v$ evaluated at $x=0$, where a jump on the initial data occurs.

The Godunov flux is given by

$$
h(u, v)= \begin{cases}\min _{[u, v]} F(w) & \text { if } u \leq v \\ \max _{[u, v]} F(w) & \text { if } u \geq v\end{cases}
$$

We remark that in the case of problem (1.1) an explicit Riemann solver at the sign change interface would require to code all the possible cases detailed in Theorem 3.1 . and would be very expensive. Aiming to have just a qualitative representation of the solution, in our computations we force $\xi\left(t^{n}\right)$ to be at the middle of the cell it belongs to at each time step, i.e. $\xi\left(t^{n}\right)=x_{j}$, for $j$ such that $\operatorname{sgn}\left(\phi_{x}\left(x_{j-1 / 2}\right)\right)>\operatorname{sgn}\left(\phi_{x}\left(x_{j+1 / 2}\right)\right)$. 
4.2.3. Rusanov scheme. In [14], the author proves convergence of EngquistOscher scheme for conservation laws with discontinuous flux. These results can be used in the second step to solve the conservation law. For our simulations we have used a variant introduced by Rusanov [13], whose flux is given by

$$
h(u, v)=\frac{1}{2}(f(u)+f(v))+\frac{1}{2} \max \left\{\left|f^{\prime}(u)\right|,\left|f^{\prime}(v)\right|\right\}(u-v)
$$

and is known to be robust.

5. Convergence results. In this section we provide some numerical tests showing the convergence of the wave-front tracking and finite volumes schemes described in the previous Sections 4.1, 4.2. To this end, we choose an initial datum $\rho_{0}$ that gives a non-trivial behavior at the turning curve. In fact, if the solution $\rho$ of 2.1 is continuous at $x=\xi(t)$, that is, $\rho(t, \xi(t)-)=0=\rho(t, \xi(t)+)$, we deal with classical solutions on each side of the turning curve, and the convergence is standard.

In the following, we consider the Riemann-type initial datum

$$
\rho_{0}(x)= \begin{cases}0 & \text { if }-1<x<0 \\ 0.9 & \text { if } 0<x<1\end{cases}
$$

and the cost function $c(\rho)=1 / v(\rho)=1 /(1-\rho)$. We run simulations up to time $T=3$, so that the whole mass lefts the domain $\Omega$ and $\rho(T, x) \equiv 0$, see Fig. 4.1.

5.1. Data processing. Wave-front tracking solutions are not defined on a standard cartesian grid in space and time, but rather as constant values on polygonal regions delimited by wave fronts (see Fig. 5.1). In order to compare the approximate solutions obtained via wave-front tracking at different mesh sizes, and finite volume approximations with the wave-front tracking reference solution, we have to convert these data sets to values defined on a reference cartesian grid.

In what follows we denote by $X_{W F T}$ the data obtained from the wave-front tracking procedure, by $\widetilde{X}_{W F T}$ the processed data and by $X_{F V}$ those obtained from finite volumes schemes. Values for $\tilde{\rho}_{W F T}^{\nu}$ are obtained by taking the corresponding values of $\rho_{W F T}^{\nu}$ at the cartesian grid nodes, see Fig. 5.1. Finally, we operate a $\mathbf{L}^{\mathbf{1}}$-norm comparison, according to the following formula :

$$
\operatorname{Err}(\nu, \Delta x)=\int_{0}^{T} \int_{-1}^{1}\left|\tilde{\rho}_{W F T}^{\nu}-\rho_{F V}\right| d x d t=\sum_{n=1}^{N} \sum_{j=1}^{J}\left|\left(\tilde{\rho}_{W F T}^{\nu}\right)_{j}^{n}-\left(\rho_{F V}\right)_{j}^{n}\right| \Delta x \Delta t^{n},
$$

where $\nu$ refers to the wave-front tracking mesh size and $\Delta x$ denotes the space-mesh size of the cartesian grid used for the finite volume scheme.

We remark that the above procedure introduces an approximation error in the computation of the $\mathbf{L}^{\mathbf{1}}$-error, since

$$
\int_{0}^{T} \int_{-1}^{1}\left|\tilde{\rho}_{W F T}^{\nu}-\rho_{F V}\right| d x d t \neq \int_{0}^{T} \int_{-1}^{1}\left|\rho_{W F T}^{\nu}-\rho_{F V}\right| d x d t
$$

5.2. Convergence of Wave Front Tracking. The theoretical proof of existence and uniqueness of solutions of (2.1) by means of the wave-front tracking method is currently under investigation. Meanwhile, we can produce numerical evidence of the 


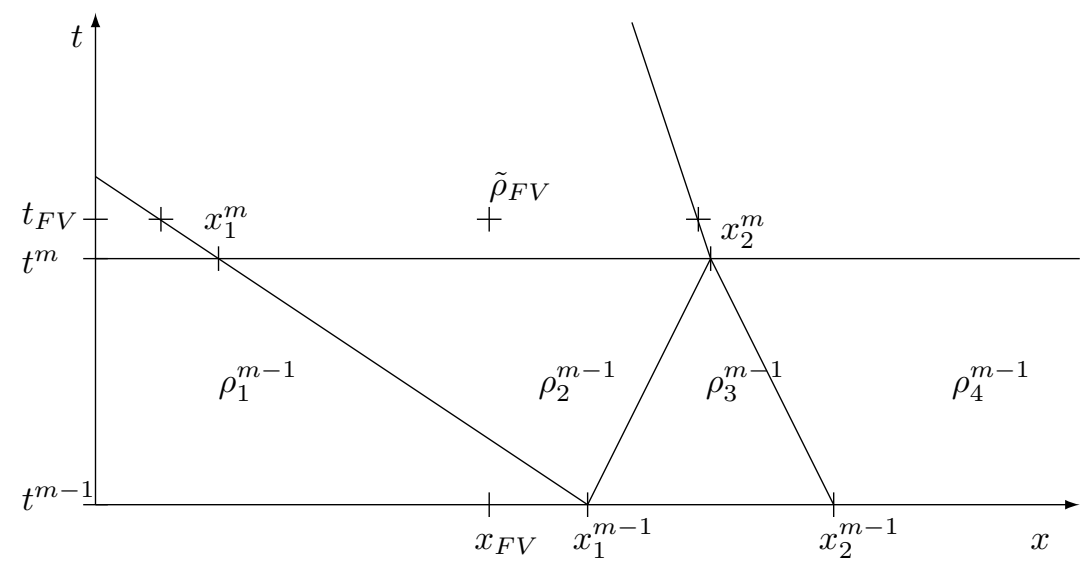

FIG. 5.1. Definition of $\tilde{\rho}_{W F T}$ at the point $\left(t_{F V}, x_{F V}\right)$ of the reference cartesian grid: after finding the last interaction time $t^{m} \leq t_{V F}$ in $\rho_{W F T}$, one has to compare the positions of the corresponding interfaces $x_{i}\left(t_{F V}\right)=x_{i}^{m}+s_{i}\left(t_{F V}-t^{m}\right)$ with respect to $x_{F V}$, where $s_{i}=1-\rho_{i}-\rho_{i+1}$ are the speeds of the wave-fronts. Here $\tilde{\rho}_{W F T}=\rho_{2}^{m}=\rho_{2}^{m-1}$.

convergence of WFT scheme by showing that the $\mathbf{L}^{\mathbf{1}}$-error between two subsequent discretization meshes $2^{-\nu}$ and $2^{-\nu-1}$

$$
\epsilon_{\nu}=\left\|\tilde{\rho}_{W F T}^{\nu+1}-\tilde{\rho}_{W F T}^{\nu}\right\|_{\mathbf{L}^{1}}
$$

decreases to zero as $\nu$ increases. Table 5.1 and Fig. 5.2 show that the sequence $\left\{\tilde{\rho}_{W F T}^{\nu}\right\}$ is a Cauchy sequence in $\mathbf{L}^{\mathbf{1}}$.

\begin{tabular}{|r|l|l|}
\hline$\nu$ & $\Delta \rho$ & $\epsilon_{\nu}$ \\
\hline 5 & $2^{-5}$ & $4.280 e-2$ \\
6 & $2^{-6}$ & $2.164 e-2$ \\
7 & $2^{-7}$ & $6.141 e-3$ \\
8 & $2^{-8}$ & $5.048 e-3$ \\
9 & $2^{-9}$ & $1.755 e-3$ \\
10 & $2^{-10}$ & $2.091 e-3$ \\
11 & $2^{-11}$ & $4.305 e-4$ \\
12 & $2^{-12}$ & $4.347 e-4$ \\
\hline \multicolumn{2}{|c}{ TABLE 5.1}
\end{tabular}

$\mathbf{L}^{\mathbf{1}}$-error $\epsilon_{\nu}$ for wave-front tracking method between two subsequent discretization meshes $2^{-\nu}$ and $2^{-\nu-1}$. The comparison is done on a cartesian grid with $\Delta x=10^{-3}$ and $\Delta t=0.5 \Delta x$.

5.3. Convergence of finite volume schemes. Once assessed the convergence of the wave-front tracking scheme, we take as reference "exact" solution the one obtained with density mesh size $\varepsilon=2^{-\nu}=2^{-10}$, and we compare it to the approximations computed by Godunov and Rusanov schemes for different space mesh sizes, computing the $\mathbf{L}^{\mathbf{1}}$-norm of the difference. The following Table 5.1 and Fig. 5.3 report the values of the $\mathbf{L}^{\mathbf{1}}$-error computed on the time interval $[0, T]$ with $T=1.2$, in order to focus on the non-classical behavior of the solution. Indeed, at $T=1.2$ vacuum has 


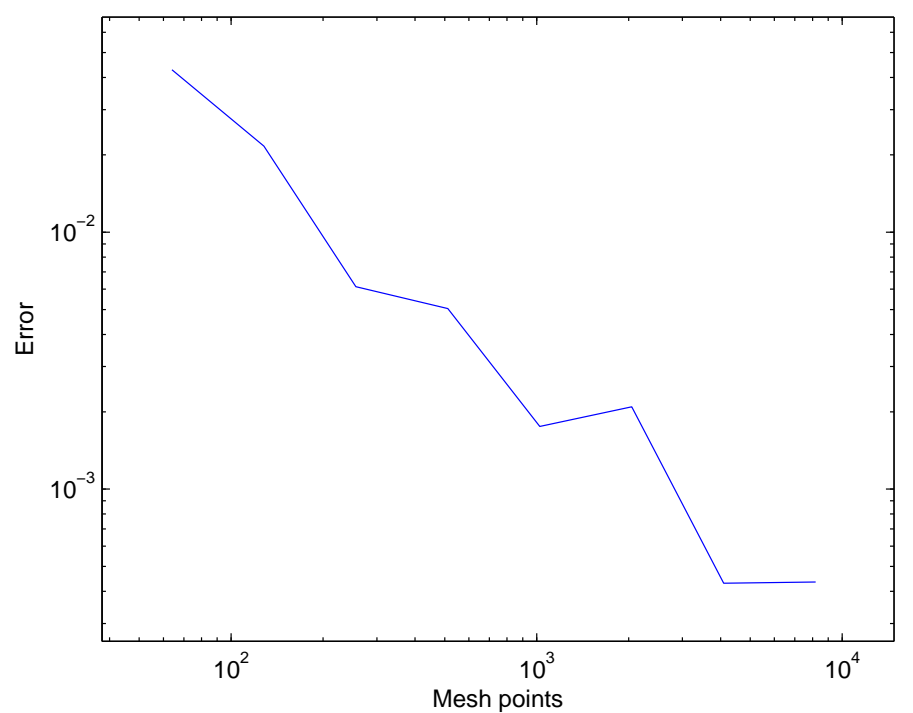

FIG. 5.2. $\mathbf{L}^{\mathbf{1}}$-error $\epsilon_{\nu}$ between two subsequent discretization meshes $2^{-\nu}$ and $2^{-\nu-1}$, for $\nu=$ $5, \ldots, 12$, corresponding to the initial datum given by 5.1 .

\begin{tabular}{|c|l|l|l|l|}
\hline$\Delta x$ & $E r r_{G}$ & $\ln \left(E r r_{G}\right) / \ln (\Delta x)$ & $E r r_{R}$ & $\ln \left(E r r_{R}\right) / \ln (\Delta x)$ \\
\hline $1 / 50$ & $7.24 e-2$ & -0.66 & $7.44 e-2$ & -0.67 \\
$1 / 100$ & $4.56 e-2$ & -0.66 & $4.68 e-2$ & -0.67 \\
$1 / 250$ & $2.49 e-2$ & -0.66 & $2.55 e-2$ & -0.67 \\
$1 / 500$ & $1.52 e-2$ & -0.67 & $1.55 e-2$ & -0.67 \\
$1 / 1000$ & $9.03 e-3$ & -0.68 & $9.12 e-2$ & -0.68 \\
$1 / 1500$ & $6.66 e-3$ & -0.69 & $6.62 e-3$ & -0.68 \\
\hline
\end{tabular}

$\mathbf{L}^{\mathbf{1}}$-norm of the error for Godunov and Rusanov schemes for 5.1 depending on the space step $\Delta x$, and corresponding convergence order. Above, $\operatorname{Err}_{G}$ stands for the error in Godunov scheme, $\mathrm{Err}_{R}$ indicates the error for Rusanov flux.

appeared around the turning point $\xi$, and the solution has become classical. For a set of space meshes ranging from $\Delta x=1 / 50$ to $\Delta x=1 / 1500$, we see a clear linear decreasing in logarithmic scale, showing numerically a convergence order of about 0.67 for both Godunov and Rusanov schemes, without an explicit treatment of the solution's behavior at $x=\xi(t)$. Fig. 5.4 shows the profiles of the three numerical approximations in a neighborhood of the turning point. We observe that Godunov scheme displays small oscillations close to $x=\xi(t)$.

5.4. More general initial data. We provide here the results of simulations performed considering a more general initial datum. We have taken

$$
\rho_{0}= \begin{cases}0.8 & \text { if }-0.8 \leq x \leq-0.5 \\ 0.6 & \text { if }-0.3 \leq x \leq 0.3 \\ 0.9 & \text { if } 0.4 \leq x \leq 0.75 \\ 0 \quad \text { elsewhere }\end{cases}
$$




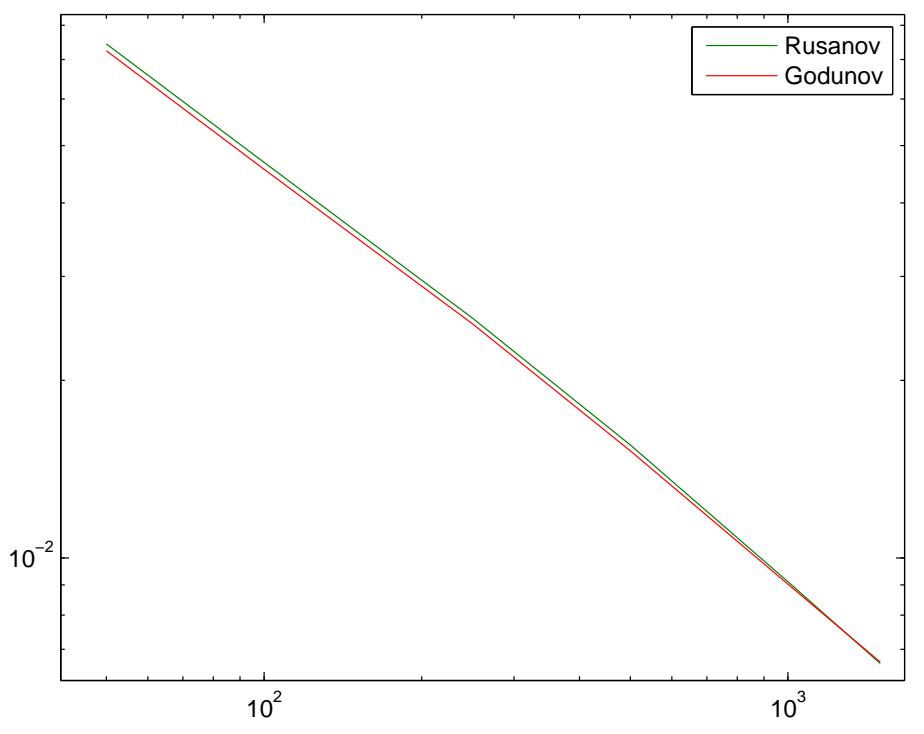

FIG. 5.3. $\mathbf{L}^{\mathbf{1}}$-norm of the error in logarithmic scale for mesh sizes $\Delta x=1 / 50, \ldots, 1 / 1500$, for Godunov and Rusanov schemes corresponding to the initial datum given by 5.1.

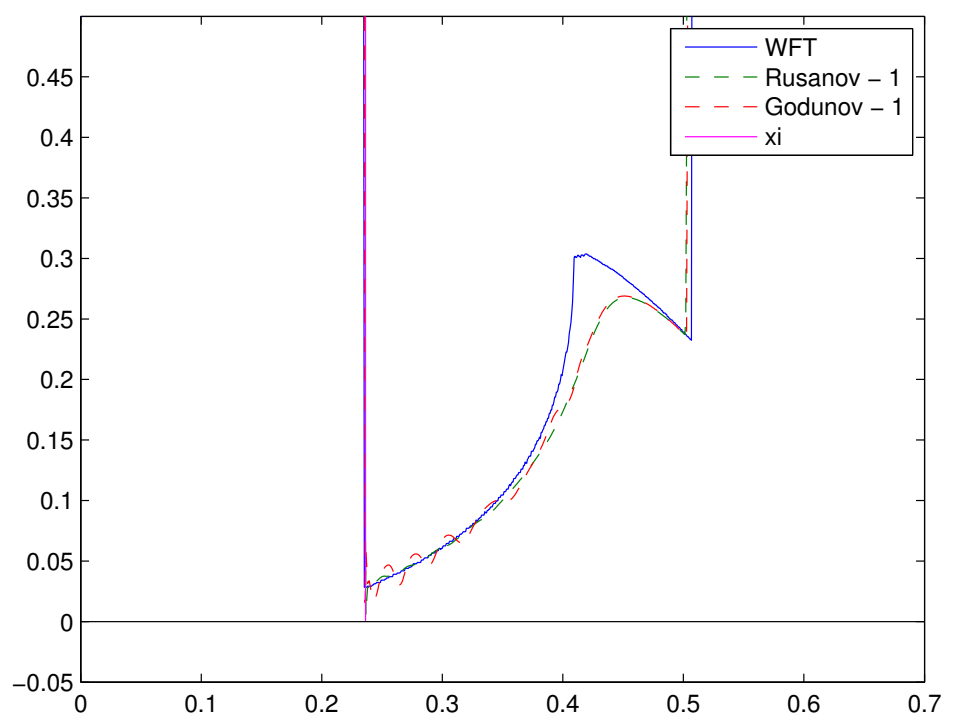

FIG. 5.4. Zoom of the numerical approximations in a neighborhood of the turning point $x=\xi(t)$ for the initial datum (5.1) at time $t=0.8$. The wave-front tracking profile is given by the mesh $\Delta \rho=2^{-10}$, and the finite volume space step is $\Delta x=1 / 1500$.

as in [4, Figure 6] in order to simulate the behavior of three mixed-density groups. 

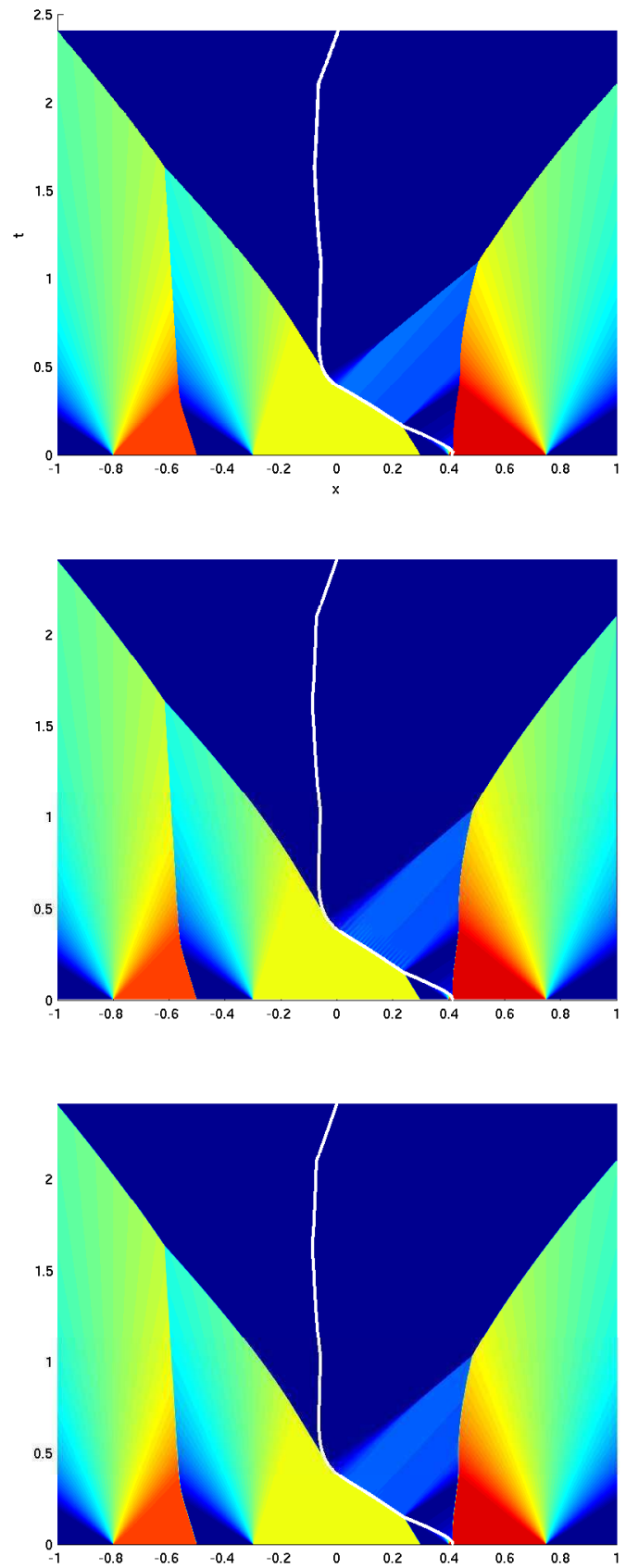

FIG. 5.5. Wave-front tracking (top), Godunov (middle) and Rusanov (bottom) schemes for $\rho_{0}$ given by 5.2. The wave-front tracking mesh is $\Delta \rho=2^{-10}$ and the finite volume space step is $\Delta x=10^{-3}$

We can see that the two finite volume simulations are evolving like the wavefront tracking one, modeling two turning phenomena. We can also observe that the 
diffusion of Rusanov counterbalances severely Godunov's oscillations.

6. Conclusions. A crowd evacuating a corridor can be modeled by a system of partial differential equations in one space-dimension, coupling a scalar conservation law describing conservation of mass and an eikonal equation assigning the direction of motion depending on the density profile. The system can be rewritten as a scalar conservation law with space-discontinuous flux function, for which the discontinuity location depends non-locally on the density profile.

Since exact entropy weak solutions cannot be computed theoretically for general initial data, we have coded an adapted wave-front tracking scheme, which is reasonably assumed to give a good approximation of problem's solutions. These wave-front tracking approximations are used as reference solutions to show numerically the convergence of classical finite volume schemes, which do not treat explicitly the dynamic at the turning point.

These results offer a sound basis to the use of classical finite volumes schemes for the computation of solutions of Hughes' model in two space dimensions, see for example [8, 11].

\section{REFERENCES}

[1] Debora Amadori and M. Di Francesco, The one-dimensional Hughes model for pedestrian flow: Riemann-type solutions, Acta Math. Sci. Ser. B Engl. Ed., 32 (2012), pp. 259-280.

[2] C. Bardos, A. Y. Le Roux, AND J.-C. NÉdÉLeC, First order quasilinear equations with boundary conditions, Comm. Partial Differential Equations, 4 (1979), pp. 1017-1034.

[3] Alberto Bressan, Hyperbolic systems of conservation laws, vol. 20 of Oxford Lecture Series in Mathematics and its Applications, Oxford University Press, Oxford, 2000. The onedimensional Cauchy problem.

[4] Marco Di Francesco, Peter A. Markowich, Jan-Frederik Pietschmann, and MarieTherese Wolfram, On the Hughes' model for pedestrian flow: the one-dimensional case, J. Differential Equations, 250 (2011), pp. 1334-1362.

[5] Nader El-Khatib, Paola Goatin, and Massimiliano D. Rosini, On entropy weak solutions of Hughes' model for pedestrian motion, Z. Angew. Math. Phys., (2012).

[6] S. K. Godunov, A difference method for numerical calculation of discontinuous solutions of the equations of hydrodynamics, Mat. Sb. (N.S.), 47 (89) (1959), pp. 271-306.

[7] Helge Holden and Nils Henrik Risebro, Front tracking for hyperbolic conservation laws, vol. 152 of Applied Mathematical Sciences, Springer-Verlag, New York, 2002.

[8] Ling Huang, S. C. Wong, Mengping Zhang, Chi-Wang Shu, and William H. K. Lam, Revisiting Hughes' dynamic continuum model for pedestrian flow and the developement of an efficient solution algorithm, Transpn. Res.-B, 43 (2009), pp. 127-141.

[9] Roger L. Hughes, A continuum theory for the flow of pedestrians, Transpn. Res.-B, 36 (2002), pp. 507-535.

[10] - The flow of human crowds, in Annual review of fluid mechanics, Vol. 35, vol. 35 of Annu. Rev. Fluid Mech., Annual Reviews, Palo Alto, CA, 2003, pp. 169-182.

[11] Yanqun Jiang, Tao Xiong, S. C. Wong, Chi-Wang Shu, Mengping Zhang, Peng Zhang, and William H. K. LAm, A reactive dynamic continuum user equilibrium model for bidirectional pedestrian flows, Acta Math. Sci. Ser. B Engl. Ed., 29 (2009), pp. 1541-1555.

[12] Kenneth H. Karlsen and John D. Towers, Convergence of the Lax-Friedrichs scheme and stability for conservation laws with a discontinous space-time dependent flux, Chinese Ann. Math. Ser. B, 25 (2004), pp. 287-318.

[13] V. V. Rusanov, The calculation of the interaction of non-stationary shock waves with barriers, Ž. Vyčisl. Mat. i Mat. Fiz., 1 (1961), pp. 267-279.

[14] John D. Towers, Convergence of a difference scheme for conservation laws with a discontinuous flux, J. Numer. Anal., 38 (2000), pp. 681-698.

[15] Hongkai ZhaO, A fast sweeping method for eikonal equations, Math. Comp., 74 (2005), pp. 603-627 (electronic). 